\title{
DUALITY IN A TWISTED HOMOLOGY THEORY
}

\author{
BY E. A. M. MACALPINE ${ }^{1}$
}

Communicated by E. Spanier, July 13, 1966

In this note we give definitions of twisted generalized homology and cohomology, in which the twisting is defined by spherical fibrations, and we prove that under certain conditions Poincare duality holds. The definitions are chosen such that when the fibrations are trivial, Whitehead's definitions, as given in [6], hold good. We use the notation of [6] and in the future refer to twisted generalized homology and cohomology simply as homology and cohomology and to spherical fibrations as fibrations. Full details of the work reported here will appear elsewhere.

I should like to thank Professor C. T. C. Wall for suggesting this work and for his guidance and criticism.

1. Let $X$ be a finite connected C.W. complex with base point and denote by $\Lambda$ the group ring of $\pi_{1}(X)$. Suppose (i) we have a homomorphism $w: \pi_{1}(X) \rightarrow\{ \pm 1\}$ defining a $\Lambda$-module structure $Z^{t}$ on $Z$ and (ii) there is an integer $n$ and a class $[X] \in H_{n}\left(X ; Z^{t}\right)$ such that, for all $r$, cap product with $[X]$ induces an isomorphism

$$
[X] \cap: H^{r}(X ; \Lambda) \rightarrow H_{n-r}\left(X ; \Lambda \otimes Z^{t}\right)
$$

then we say $X$ is an $n$-dimensional connected Poincaré complex. By a space $X$, we shall mean a connected Poincaré complex. We assume that for all spectra [6] a mentioned, there exists an integer $N$ such that $A_{N+i}$ is $i$-connected for all $i \geqq 0$ and we denote by $e(A)$ the map $S A_{n} \rightarrow A_{n+1}\left(\right.$ all $n$ ) where $S A_{n}$ denotes (reduced) suspension of $A_{n}$, $S^{1} \wedge A_{n}$.

Let $\xi$ be an $(r-1)$ spherical fibration [2] over a space $X$ and denote by $X^{\xi}$ its Thom space (mapping cone of the projection map). Then we define the $p$ th cohomology group of $X$, with values in a spectrum a twisted by $\xi$, as

$$
H^{p}(X ; \propto(\xi))=\lim _{\overrightarrow{\boldsymbol{\alpha}}}\left\{S^{\alpha} X^{\xi}, A_{\alpha+r+p}\right\} .
$$

$\{V, W\}$ denotes stable homotopy classes of $S$-maps of $V$ into $W$; the direct limit is over the positive integers and the connecting homomorphisms are given by suspension composed with $e(A)$. (As all our groups are reduced we write $H^{p}(X)$ for $H^{p}\left(X, x_{0}\right)$.) If $\eta$ is an $(s-1)$ -

${ }^{1}$ Work supported by S.R.C. and Edinburgh University. 
spherical fibration over $Y$ we define the $q$ th homology group of $Y$, with values in a spectrum $Q$ twisted by $\eta$, as

$$
H_{q}(Y ; Q(\eta))=\lim _{\vec{\beta}}\left\{S^{q+\beta+\beta}, A_{\beta} \wedge Y^{\eta}\right\} .
$$

If $\xi$ is trivial $X^{\xi}=S^{r} X$ and the definitions of [6] result provided, of course, the spectra are restricted as above.

2. Let $\Psi: a \otimes B \rightarrow \mathcal{C}\left(\psi: A_{p} \wedge B_{q} \rightarrow C_{p+q}\right)$ be a pairing of spectra [6]. Then we can construct all the usual products in a homology theory provided we choose the twistings carefully. In $\$ 4$ we shall construct the cap product.

3. Spivak in [4] shows that for an $n$-dimensional space $X$ (in our sense) there exists a fibration $\nu$ (fibre dimension $(r+s-1)$, say) unique up to suspension and fibre homotopy equivalence such that $\nu$ is reducible; that is, the map $j_{1}: X^{\nu} \rightarrow S^{n+r+s}$, obtained by collapsing everything outside one top cell to a point, has a right homotopy inverse $j_{2}, j_{1} \circ j_{2} \simeq 1$. From now on we assume $X$ and $\nu$ as above. Let $\Sigma$ denote the sphere spectrum; then we can show $H^{n}(X ; \Sigma(\nu)) \cong \boldsymbol{Z}$ (the argument is too long to give here) and any map $f \simeq j_{1}: X^{\nu} \rightarrow S^{n+r+s}$ represents a generator. We say an element $z \in H_{n}(X ; \Sigma(\nu))$ is a fundamental class if it can be represented by a map $g \simeq j_{2}: S^{n+r+s} \rightarrow X^{\nu}$ (alternatively, $f \circ g$ a map of degree +1 .)

4. Let $\xi$ be a fibration over $X$ (fibre dimension $(r-1)$, say); then it can be shown (using the $K$-theory for spherical fibrations) that there is a fibration $\eta$ over $X$ such that $\xi$, appropriately suspended, joined to $\eta$, appropriately suspended, is some suspension of $\nu$ (up to fibre homotopy equivalence). Since we are only working up to suspension we may consider $\nu$ as the join of $\xi$ and $\eta$, provided $r, s>n$, the dimension of $X$ [5]. Hence we take the fibre dimension of $\eta$ to be $(s-1)$.

We now follow [5, Chapter 3]. We use the notation of $\$ 3$ and let $\xi, \eta$ be as above. Consider the join fibration of $\xi$ and $\eta$ over $X \times X$, written $\xi * \eta$ (over each point $\left(x_{1}, x_{2}\right) \in X \times X$ we take the fibre to be the join of the fibre over $x_{1}$, belonging to $\xi$, and the fibre over $x_{2}$, belonging to $\eta$ ), then the diagonal map $\Delta: X \rightarrow X \times X$ induces the fibration $\nu$ over $X$ and we have the composite map

$$
U: S^{n+r+s} \stackrel{j_{2}}{\rightarrow} X^{\nu} \stackrel{\Delta_{*}}{\rightarrow}(X \times X)^{\xi * \eta}
$$

where $\Delta_{*}$ is the induced map of Thom spaces. Now by [1] $(X \times X)^{\xi * \eta}$ is homeomorphic to $X^{\xi} \wedge X^{\eta}$, so $U$ is a map $S^{n+r+s} \rightarrow X^{\xi} \wedge X^{\eta}$. Accord- 
ing to [4] $U$ is a duality map in the sense of [3] and hence $X^{\xi}, X^{\eta}$ are $(n+r+s)$-duals.

We now construct the cap product. Consider the three groups $H_{n}(X ; \Sigma(\nu)), H^{q}(X ; a(\xi)), H_{n-q}(X ; \propto(\eta))$ and let $g: S^{n+r+s+\alpha} \rightarrow S^{\alpha} X^{\nu}$, $f: S^{\alpha} X^{\xi} \rightarrow A_{\alpha+r+q}$ be representatives of elements of the first two groups respectively. Then the composite map $f^{\prime}$

$$
S^{n+r+s+\alpha} \stackrel{g}{\rightarrow} S^{\alpha} X^{\nu} \stackrel{\left(1 \wedge \Delta_{*}\right)}{\rightarrow} S^{\alpha} X^{\xi} \wedge X^{\eta} \stackrel{(f \wedge 1)}{\longrightarrow} A_{\alpha+r+q} \wedge X^{\eta}
$$

represents an element of $H_{n-q}(X ; \mathfrak{Q}(\eta))$. By checking that this map $f^{\prime}$ commutes with the connecting homomorphisms involved, we have the cap product

$$
\cap: H_{n}(X ; \Sigma(\nu)) \otimes H^{q}(X ; \propto(\xi)) \rightarrow H_{n-q}(X ; Q(\eta)) .
$$

5. We can now state the

Theorem. Let $X$ be an $n$-dimensional connected Poincarê complex. Then there exists a fundamental class $z \in H_{n}(X ; \Sigma(\nu))$ and

$$
z \cap: H^{q}(X ; Q(\xi)) \cong H_{n-q}(X ; \propto(\eta)) .
$$

Sketch of Proof. The existence of $z$ was established in $\S 3$. By adapting Spanier's work [3] (see Corollary (6.9)) to the case of duality maps $U^{\prime}: S^{n+r+s} \rightarrow Y^{\prime} \wedge Y$ we can prove that for $V, W, Y^{\prime}, Y$ finite C.W. complexes there is an isomorphism

$$
\Gamma:\left\{V \wedge Y, S^{n+r+s} W\right\} \rightarrow\left\{V, W \wedge V^{\prime}\right\}
$$

where a representative $f$ of an element of the left hand side corresponds to a representative $f^{\prime},\left\{f^{\prime}\right\}=\Gamma\{f\}$, under the map

$$
S^{n+r+s} V \stackrel{\left(U^{\prime} \wedge 1\right)}{\longrightarrow} Y^{\prime} \wedge Y \wedge V \leftrightarrow V \wedge Y \wedge Y^{\prime} \stackrel{(f \wedge 1)}{\longrightarrow} S^{n+r+s} W \wedge Y^{\prime} .
$$

The middle map, $\leftrightarrow$, is the homotopy equivalence obtained by switching factors. By applying this to the duality map $U$ of $\S 4$ we have

$$
\left\{S^{\alpha} X^{\xi}, A_{\alpha+r+q}\right\} \cong\left\{S^{n+r+s+\alpha}, A_{\alpha+r+q} \wedge X^{\eta}\right\} .
$$

The isomorphism given by $\Gamma$ commutes with the connecting homomorphisms of the direct limits and so

$$
H^{q}(X ; \mathfrak{Q}(\xi)) \cong H_{n-q}(X ; \mathfrak{Q}(\eta)) .
$$

It is now easy to check that the composite map giving the cap product and that giving $\Gamma$ agree up to homotopy.

6. It is hoped eventually to extend the basic definitions to cover 
more general twistings, possibly involving fibrations over $X$ with fibre $A_{\alpha}$.

\section{REFERENCES}

1. M. F. Atiyah, Thom complexes, Proc. London Math. Soc. 11 (1961), 291-310.

2. J. W. Milnor, Characteristic classes for spherical fibre spaces, (to appear).

3. E. H. Spanier, Function spaces and duality, Ann. of Math. 70 (1959), 338-378.

4. M. Spivak, On spaces satisfying Poincaré duality, Ph.D. Thesis, Princeton University, Princeton, N. J., 1964.

5. C. T. C. Wall, Poincaré complexes. I, (to appear).

6. G. W. Whitehead, Generalized homology theories, Trans. Amer. Math. Soc. 102 (1962), 227-283.

Department of Pure Mathematics,

THE UNIVERSITY, LIVERPOOL 